\title{
Conditioned suppression in the rat as a function of shock reinforcement schedule'
}

\author{
RICHARD D. WILLIS, UNIVERSITY OF ILLINOIS \\ ROBERT W. LUNDIN, THE UNIVERSITY OF THE SOUTH
}

The effects of shock-reinforcement schedules in conditioned suppression were studied in three male albino rats. $S_{2}$ (electric shock) was paired randomly with the $S_{1}$ either $10 \%, 50 \%$, or $90 \%$ of the time. Each presentation was correlated with a different $S_{I}$ (tone, clicker, light). Each $S$ received all three shock reinforcement schedules. Then the $S_{2} s$ were presented randomly by days, each with its appropriate $S_{1}$ until each $S_{1}$ had been presented fifty times in this way. Suppression ratios varied monotonically with shockreinforcement schedule, with the greatest suppression at $90 \%$. When the $S_{2} s$ were presented randomly by days, the greatest variability occurred with the $50 \%$ schedule. The results are interpreted in terms of superstitious avoidance behavior.

Estes \& Skinner (1941) were the first to publish results on conditioned suppression. Since then there have been several studies of the phenomenon, but few have investigated the effect of pairing the aversive stimulus with a pre-aversive stimulus on an intermittent basis. Geller \&ailan, Stein, \& Brady (1957) conditioned three groups of rats each on a $100 \%, 50 \%$, and $25 \%$ shock-reinforcement schedule. They found no difference among the groups in rate of acquisition of the suppression response, but the groups conditioned on the partial schedules were slower to extinguish.

The present experiment was designed to study the effect of partial shock-reinforcement in a within-S design.

Subjects

The Ss were three Carworth CFN male albino rats approximately 160 days old at the beginning of the experiment. Throughout the experiment they were maintained at $80 \%$ of the pre-experimental body weight. Apparafus

A standard commercial chamber and pellet feeder, mounted in a picnic ice box was used. Reinforcement was a $45 \mathrm{mg}$ pellet. $\mathrm{S}_{1}$ 's were a light, a click, and a tone. $\mathrm{S}_{2}$ was a scrambled 2 sec. 1 ma DC shock.Water was available during each session. The experimental chamber was kept dark except when the $S_{1}$ was a light. Bar-press responses were recorded on counters and a cumulative recorder; the number of responses in each $\mathrm{S}_{1}$ presentation was recorded on a print-out counter.

\section{Procedure}

Experimental sessions were run daily at the same time. All three Ss were conditioned to bar press with continuous reinforcement and then switched to a
VI: 1 min. schedule. After this VI responding had stabilized, suppression conditioning was begun. Each $S$ was given 60 conditioning trials ( $S_{1}$ presentations) at one shock percentage, then switched to a different $\mathrm{S}_{1}$ and a different shock percentage for 60 more trials, and finally switched to a third $S_{1}$ and shock percentage for 60 more trials. These orders were as follows:

S1: tone $90 \%$, clicker $10 \%$, light $50 \%$

S2: tone $10 \%$, light $90 \%$, clicker $50 \%$

S3: tone $50 \%$, clicker $90 \%$, light $10 \%$

After the 180 conditioning trials, the presentation of $\mathrm{S}_{1}$ 's and correlated shock percentages were randomized by days. This procedure was continued until each $S$ had received 50 presentations of each type of $S_{1}$. Throughout the experiment the duration of $S_{1}$ was $4 \mathrm{~min}$. and the time between the termination of $S_{1}$ on one trial and the presentation of the next $s_{1}$ was randomly varied from 4 to $30 \mathrm{~min}$.

The dependent variable, computed for each $\mathrm{S}_{1}$ presentation was response rate during $S_{1}$ minus baseline rate, divided by baseline rate. (Baseline rate was rate in absence of $\mathrm{S}_{1}$ computed for each session.) A suppression ratio of -1.00 indicated that no responses were made in the presence of the $s_{1}$; a suppression ratio of 0.00 indicated response rate during $S_{1}$ equalled the baseline rate. To estimate the variability of the baseline at the end of the experiment, 50 intervals of $4 \mathrm{~min}$. without $\mathrm{S}_{1}$ were sampled and a pseudosuppression ratio computed for each; i.e., the ratio

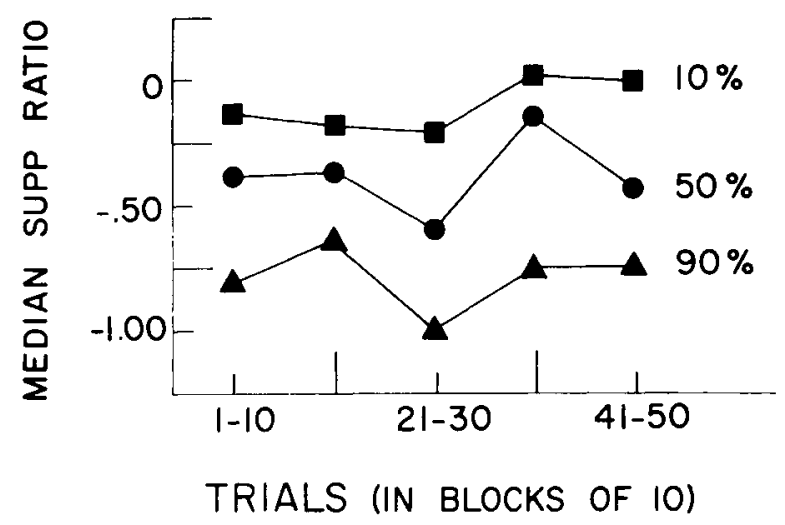

Fig. 1. Median suppression ratio for 3 animals as a function of trials (in blocks of 10) in the final stage of experimentation at $\mathbf{1 0} \%, 50 \%$, and $\mathbf{9 0} \%$ pairing of the $\mathrm{S}_{2}$ with $\mathrm{s}_{1}$. Each plotted point is the median of thirty observations. 


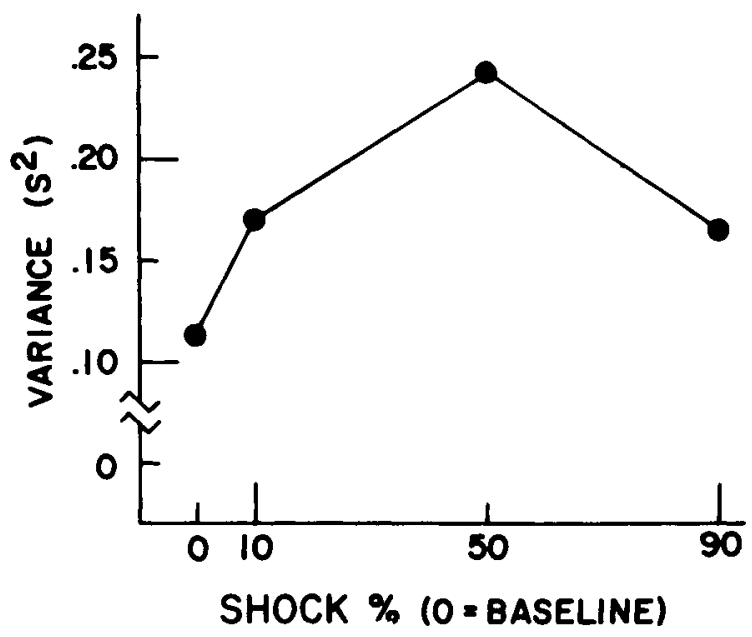

Fig. 2. Variability of responding (variance of suppression ratios) in the final stage of experimentation at $0 \%$ (baseline), $10 \%, 50 \%$, and $90 \%$ pairing of $s_{2}$ with $s_{1}$ for 3 animals. Variances are based on 150 observations at each value.

of the difference between the response rate in the sampled 4-min. interval and the baseline rate over the baseline rate.

Results

Figure 1 shows the median suppression ratios (across Ss) in blocks of 10 trials under the terminal conditions. The data for mean suppression ratios were almost identical. There is a monotonic relation between $S_{2}$ percentage and median suppression ratio, with the higher percentages related to greater suppression. The Friedman two-way analysis of variance by ranks (Siegel, 1956) was used to estimate the significance of difference between medians under the four conditions (Baseline, 10\%,50\%, and 90\%). The obtained probability was $\left(\mathrm{X}^{2} \mathrm{r}=8.2, \mathrm{~J}=4, \mathrm{~K}=3, \mathrm{p}=.017\right)$. The type of $\mathrm{S}_{1}$ had no effect on the median suppression ratio $\left(\mathrm{X}^{2} r=0.00, \mathrm{~J}=3, \mathrm{~K}=3, \mathrm{p}=1.00\right)$. The difference in median suppression ratios due to Ss was also not significant $\left(\mathrm{X}^{2} \mathrm{r}=1.50, \mathrm{~J}=3, \mathrm{~K}=4, \mathrm{p}=.653\right)$.

Figure 1 indicates that the median suppression ratios were stable over time at each schedule shock percentage. However, the shock schedule appeared to affect re- sponse variability as seen in Fig. 2. A Friedman analysis of the difference in variance of suppression ratios due to the different shock schedules did not indicate any significant differences: $\left(\mathrm{X}^{2}{ }_{r}=5.8, \mathrm{~J}=4\right.$, $\mathrm{K}=3, \mathrm{p}=.18$ ).

Discussion

These data are consistent with the findings of Geller, Kailan, Stein, \& Brady (1957) in that there is less suppression with the more intermittent shock pairings. Although the differences in variance of suppression ratios due to shock reinforcement schedules were not significant, the trend toward greater response variability in the $50 \%$ schedule suggests the possibility that superstitious avoidance behavior is operating. A response can be considered avoidance if it occurs at the end of the $S_{1}$ interval which is terminated without the presentation of $S_{2}$. If such behavior is maintained by scheduled shocks, it could be considered superstitious in that the stimulus events are not, in fact, responsecontingent.

At the $50 \%$ schedule, the median suppression ratio was stable over time (Fig. 1) but tended to show more variability than under the other conditions (Fig. 2). At $50 \%$ random pairings there were blocks of $\mathrm{S}_{1}$ presentations which were not followed by shock. In these intervals, it is possible that the organism was superstitiously learning an avoidance response. However, these trials would be followed by other trials in which the $S_{2}$ was presented, and so the avoidance behavior would extinguish. These would be followed by non-shocked trials, during which further superstitious avoidance behavior would occur.

\section{References}

Estes, W. K., \& Skinner, B. F. Some quantitative properties of anxiety. J. exp. Psychol., 1941, 29, 390-400.

Geller, I., Kailan, H., Stein, L., \& Brady, J. V. Acquisition and extinction of conditioned "fear" as a function of partial shock reinforcement. Paper presented at the meetings of the Eastern Psychological Association, April, 1957.

Siegel, S. Nonparametric statistics for the behavioral sciences. New York: McGraw-Hill, 1956.

\section{Note}

1. The research for this paper was conducted while both authors were at Hamilton College. Acknowledgement is hereby given to Dr. C. A. Godcharles for his cooperation in this project. 